\title{
Diagnósticos de enfermagem em idosos institucionalizados e a associação com o processo de incapacidade funcional
}

\author{
Simone Cristina de Souza*, Renato Gorga Bandeira de Mello**, Patricia Morsch ${ }^{\star * *}$, \\ Cibele de Souza Prates ${ }^{* * * *}$, Gustavo Nunes Pereira ${ }^{* * * *}$
}

\section{Resumo}

O objetivo principal deste estudo foi investigar os diagnósticos de enfermagem mais prevalentes em uma instituição de longa permanência para idosos e suas associações com a incapacidade funcional. A pesquisa caracteriza-se como documental, com abordagem quantitativa. Foi realizada a revisão de prontuários de todos os idosos, que residiram ou residem na instituição, de junho de 2008 até março de 2013, totalizando 120 indivíduos. A partir de então, foram observados e descritos os principais diagnósticos de enfermagem e comorbidades clínicas, tendo sido realizada uma associação dos diagnósticos encontrados com o desfecho de incapacidade funcional, classificada por meio do índice de Katz. Foi encontrada uma associação positiva entre demência e dependência $(p<0,001)$. Na análise multivariada foi possível demonstrar que demência é fator de risco independente para perda de funcionalidade e a presença do desfecho é $28 \%$ maior nos idosos com demência quando comparados àqueles sem essa condição $(p<0,001)$. A variável idade também foi identificada de forma significativa $(p=0,05)$ como fator de risco independente para perda de funcionalidade. Conclui-se, portanto que variáveis identificadas na avaliação de enfermagem podem predizer incapacidade funcional em idosos institucionalizados.

Palavras-chave: Geriatria. ILPI. Serviços de saúde para idosos. Incapacidade. Diagnóstico de enfermagem.

\section{Introdução}

Segundo o censo brasileiro de 2010, a população de 60 anos ou mais de idade era de 20,6 milhões de pessoas, contra 14,5 milhões em 2000 e 10,7 milhões em 1991. A proporção de idosos na década de 1990 era de $7,3 \%$, contra $8,6 \%$ em 2000 e $10,8 \%$ em 2010. Nesse período, por conseguinte, o número de idosos aumentou em quase dez milhões, resultado do crescimento vegetativo e do aumento gradual da esperança média de vida (IBGE, 2012).

* Faculdade de Enfermagem. Centro Universitário Metodista do Sul - IPA. Brasil. E-mail: residencialconvivencia@ gmail.com

** Instituto de Geriatria e Gerontologia - PUCRS. E-mail: renatogbmello@gmail.com

**** Instituto de Geriatria e Gerontologia - PUCRS. E-mail: patriciamorsch@hotmail.com

***** Faculdade de Enfermagem. Centro Universitário Metodista do Sul - IPA. E-mail: cibeleprates@yahoo.com.br

***** Instituto de Geriatria e Gerontologia - PUCRS. E-mail: gustavojaguarao@hotmail.com

$\hookrightarrow$ http://dx.doi.org/10.5335/rbceh.2014.4061

Recebido em: 17/11/2014. Aceito em: 07/03/2015 
Além da transição demográfica descrita, houve um processo de transição epidemiológica com consequente aumento na prevalência de doenças não transmissíveis, sobretudo cardiovasculares e neuropsiquiátricas, que agregam significativa morbidade e limitações funcionais aos idosos (BRASIL, 2011; SCHMIDT et al., 2011).

Inicialmente, alguns autores definiram a incapacidade funcional como a dificuldade experimentada em realizar atividades em qualquer domínio da vida, devido a um problema físico ou de saúde, com impactos sobre a habilidade para exercer papéis e atividades na sociedade. Essas atividades são agrupadas em atividades básicas de vida diária (ABVDs) - que caracterizam a habilidade para realizar tarefas de autocuidado (banhar-se, vestir-se, ir ao banheiro, ser continente e alimentar-se) e de mobilidade (transferência). As ABVDs são hierarquicamente relacionadas, e a perda da capacidade na sua realização se dá nas atividades mais complexas, como vestir-se, até as de autorregulação, como alimentar-se (KATZ et al., 1963; BARBOSA et al., 2014).

Tais limitações funcionais demandam cuidados especiais, sobretudo de equipes multiprofissionais com capacitação em geriatria e gerontologia. Nesse cenário, a longevidade é contraditória, porque, ao mesmo tempo em que significa mais anos de vida, implica também mais prejuízos físicos e psicológicos, acarretados principalmente pelas doenças crônicas não transmissíveis (MEIRA et al., 2013; BRASIL, 2011; CAMARANO, 2010). Para fornecer esse tipo de atendimento, instituições de longa per- manência para idosos (ILPIs) são uma alternativa ao cuidado do idoso, principalmente daqueles com maiores graus de dependência, apesar de, no Brasil, o cuidado familiar ainda ser preponderante (PASINATO; KORNIS, 2010).

A enfermagem, como responsável pela estruturação do conhecimento das respostas humanas aos problemas de saúde tem o intuito de proporcionar ao indivíduo as melhores condições de saúde. O enfermeiro, para descrever e desenvolver um plano de cuidados fundamentado cientificamente e com confiança, utiliza-se da identificação dos diagnósticos de enfermagem (CARPENITO-MOYET, 2011).

Considerando os aspectos referidos de transição demográfica e epidemiológica, o aumento da necessidade de cuidados de idosos em instituições, faz-se necessário o conhecimento de variáveis preditoras de perdas funcionais para que sejam identificadas e possam auxiliar o planejamento dos cuidados de enfermagem e da equipe multiprofissional. Porém, o perfil de diagnósticos e suas associações com limitações funcionais nessa população, especialmente em uma ILPI privada, têm sido pouco descritas.

Diante disso, o presente estudo teve por objetivo investigar a prevalência de diagnósticos de enfermagem em idosos institucionalizados e suas associações com a incapacidade funcional.

\section{Métodos}

Trata-se de uma pesquisa documental seguindo uma abordagem quantitativa e descritiva. A ILPI na qual os 
dados foram coletados está localizada na cidade de Porto Alegre, Rio Grande do Sul, e caracteriza-se por prestar serviço multiprofissional especializado a idosos, na sua maioria com limitações funcionais e cognitivas que geram dependência. A instituição conta, atualmente, com 42 idosos residentes, alocados de acordo com as características do paciente e a necessidade de cuidados específicos.

Foram incluídos na análise dados de prontuário de 120 indivíduos que residiram ou residem na instituição, com 60 anos ou mais, sendo excluídos aqueles cujos prontuários não apresentavam dados suficientes para compor as variáveis de interesse. O período avaliado foi de junho de 2008 até março de 2013. Os dados analisados foram extraídos por meio de uma ficha de coleta de dados e armazenados em planilha do MS Excel.

Com base em prevalências descritas na literatura, foram definidos enfoques específicos inseridos em grupos como: a) nutrição desequilibrada, menor que a necessidade corporal (risco de ingestão de nutrientes acima ou abaixo das necessidades metabólicas), deglutição prejudicada (funcionamento anormal do mecanismo de deglutição associado a déficit na estrutura ou na função oral, faríngea ou esofágica); b) eliminações: constipação (diminuição na frequência normal de evacuação, acompanhada por passagem de fezes difícil ou incompleta e/ou eliminações de fezes excessivamente duras e secas), incontinência urinária e fecal; c) atividade e repouso: deambulação prejudicada (limitação dos movimentos independentes, a pé, pelo ambiente), padrão de sono perturbado (interrupções na quantidade e da qualidade do sono, limitadas pelo tempo, decorrentes de fatores externos), e) presença de quadro demencial e ansiedade (vago e incômodo sentimento de desconforto ou temor, acompanhado por resposta autonômica, sentimento de apreensão causada pela antecipação de perigo; é um sinal de alerta que chama a atenção para um perigo iminente e permite ao indivíduo tomar medidas para lidar com ameaça) (NANDA, 2012). Também foi realizado um levantamento, a fim de observar as principais comorbidades clínicas encontradas nessa população, assim como suas prevalências.

Após essa etapa, foi realizada uma associação dos diagnósticos de enfermagem encontrados com o desfecho incapacidade funcional para atividades básicas, classificada por meio do índice de Katz (KATZ et al., 1963). As atividades analisadas foram: banhar-se, vestir-se, ir ao banheiro, comer, deitar-se e levantar-se da cama/cadeira e ser continente nas funções de urinar/evacuar.

A incapacidade funcional para atividades básicas foi definida como: necessidade de ajuda parcial ou total para, no mínimo, uma das atividades diárias investigadas. Assim, o índice de Katz foi dicotomizado em: zero (independente para todas as atividades) e um (dependente para uma ou mais atividades).

$\mathrm{Na}$ análise estatística, as variáveis categóricas foram descritas em porcentagem e as associações foram aferidas por meio do teste Qui-Quadrado. Já as variáveis contínuas foram descritas em média e desvio padrão. As associações independentes entre os fatores em estudo 
e o desfecho funcionalidade foram verificadas por meio de regressão de Poisson, modificada por um modelo hierarquizado. A regressão de Poisson é uma forma de análise de regressão usada para modelar contagem de dados e tabelas de contingência.

Intervalos de confiança acima de um foram considerados significativamente risco para o desfecho e quando abaixo de um, foram considerados fatores de proteção para o desfecho. As análises foram conduzidas no software Statistical Program for Social Sciences (SPSS ${ }^{\oplus}$ software, versão 17.0, Chicago, IL).

Este estudo respeitou os preceitos éticos, seguindo as diretrizes e normas de pesquisa que envolvem seres humanos prevista na Resolução 466/2012, e foi aprovado pelo Comitê de Ética em pesquisa do Centro Universitário Metodista IPA, conforme o número de protocolo 280/2012. Assim, foi documentada a autorização da instituição por meio de um termo de aceitação de realização do estudo.

\section{Resultados}

A idade dos 120 idosos incluídos nesta análise variou de 63 a 107 anos, com média de 85,2 anos $( \pm 8,4), 64,2 \%$ da amostra pertencia ao sexo feminino, com idade média de 85,2 anos $( \pm 8,7)$. Já do sexo masculino, a idade média foi de 85,1 anos $( \pm 7,8)$. A distribuição dos idosos quanto às características sociodemográficas encontra-se na Tabela 1, em que se observa que a maioria é mulheres com mais de 80 anos de idade e viúvas.
Tabela 1: Características sociodemográficas dos idosos institucionalizados

\begin{tabular}{l|r|r}
\cline { 2 - 3 } & \multicolumn{1}{c|}{$\mathrm{n}$} & \multicolumn{1}{c}{$\%$} \\
\hline Sexo & 43 & 35,8 \\
Masculino & 77 & 64,2 \\
Feminino & & \\
Faixa etária & 9 & 7,5 \\
60-69 anos & 11 & 9,2 \\
70-79 anos & 63 & 52,5 \\
80-89 anos & 37 & 30,8 \\
90 anos ou mais & & \\
Escolaridade & 16 & 13,3 \\
Nunca estudou & 50 & 41,7 \\
1-4 anos de estudo & 32 & 26,7 \\
5-8 anos de estudo & 22 & 18,3 \\
9 anos ou mais & & \\
Situação conjugal & 13 & 10,8 \\
Solteiro(a) & 25,9 \\
Casado(a) & & 1,6 \\
Separado(a) & & 61,7 \\
\hline Viúvo(a) & & \\
\hline
\end{tabular}

Fonte: dados da pesquisa, 2013.

Em relação aos diagnósticos de enfermagem, alterações em aspectos nutricionais, de locomoção e de capacidade cognitiva foram prevalentes na amostra estudada e estão descritos na Tabela 2. Alta frequência de diagnósticos clínicos foi identificada na amostra estudada, destacando-se elevada prevalência de incontinências esfincterianas, história prévia de acidente vascular cerebral (AVC) e doenças cardiopulmonares são apresentadas na Tabela 3. 
Tabela 2: Diagnósticos de enfermagem relacionados aos idosos institucionalizados
Tabela 3: Comorbidades presentes nos idosos institucionalizados

Nutrição desequilib
Sim
Via para nutrição
Oral
Oral com auxílio
Enteral + via oral
Enteral exclusiva

Deglutição prejudicada

Sim

Constipação

Sim

Funcionalidade

Independentes

Deambulação

Independente

Com auxílio

Acamado

Padrão de sono prejudicado

Sim

Síndrome demencial

Sim

Ansiedade

Sim

Fonte: dados da pesquisa, 2013.

$n$
$\mathrm{n}$

30

\begin{tabular}{l|l}
$\mathrm{n}$ & $\%$ \\
\hline
\end{tabular}

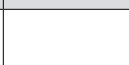

36,7

$$
25
$$

20,9

\begin{tabular}{l|c|c}
\cline { 2 - 3 } & $\mathrm{n}$ & $\%$ \\
\hline Tabagismo prévio & 28 & 18,4 \\
Sim & & \\
Etilismo prévio & 17 & 14,0
\end{tabular}

Sim

Obesidade

Sim

Desnutrição

Sim

Incontinência urinária

Sim

Incontinência fecal

Sim

Hipertensão arterial sistêmica

Sim

Diabetes melittus (DM)

Sim

Internação no último ano

Sim

\section{1}

34,2

Acidente vascular encefálico

(AVE) prévio

Sim

10,8

Diagnóst Sim

Insuficier

Sim

Doença pulmonar obstrutiva crônica

\section{9}

49,2

43

35,8

Infecção respiratória recorrente

Sim

Câncer prévio

Sim

37,5

Osteoart

cional

Sim

Osteop

57,5

Fonte: dados da pesquisa, 2013.
A Tabela 4 apresenta comparação de prevalência de incapacidade funcional para ABVDs entre diferentes variáveis sociodemográficas e clínicas com o intuito de selecionar variáveis de relevância para 0 modelo de análise multivariada. Após tais análises, foi possível avaliar a associação 
de diagnósticos de enfermagem com incapacidade funcional de forma independente de possíveis fatores de confusão (Tabela 5).

Tabela 4: Prevalência de perda de funcionalidade de acordo com características sociodemográficas e comorbidades dos idosos

\begin{tabular}{|c|c|c|}
\hline & & \\
\hline & $\%$ & Valor $\mathrm{P}$ \\
\hline Idade & & 0,4 \\
\hline$<80$ anos & 85,0 & \\
\hline$\geq 80$ anos & 90,0 & \\
\hline Sexo & & 0,7 \\
\hline Masculino & 88,3 & \\
\hline Feminino & 90,7 & \\
\hline Escolaridade & & 0,08 \\
\hline Nunca estudou & 81,3 & \\
\hline 1-4 anos de estudo & 90,0 & \\
\hline 5-8 anos de estudo & 84,4 & \\
\hline 9 anos ou mais & 100,0 & \\
\hline Obesidade & & 0,4 \\
\hline Não & 90,3 & \\
\hline Sim & 85,2 & \\
\hline Desnutrição & & 0,7 \\
\hline Não & 90,0 & \\
\hline Sim & 86,7 & \\
\hline Hipertensão arterial & & 0,4 \\
\hline Não & 86,0 & \\
\hline Sim & 91,4 & \\
\hline Insuficiência cardíaca & & 0,3 \\
\hline Não & 86,6 & \\
\hline Sim & 92,5 & \\
\hline Diabetes mellitus & & 0,04 \\
\hline Não & 93,1 & \\
\hline Sim & 78,8 & \\
\hline DPOC & & 0,3 \\
\hline Não & 90,4 & \\
\hline Sim & 81,3 & \\
\hline Depressão & & 0,04 \\
\hline Não & 93,1 & \\
\hline Sim & 78,8 & \\
\hline AVE prévio & & 0,04 \\
\hline Não & 93,1 & \\
\hline Sim & 78,8 & \\
\hline Infecção respiratória & & 0,1 \\
\hline Não & 85,3 & \\
\hline Sim & 94,2 & \\
\hline Ansiedade & & 0,002 \\
\hline Não & 97,1 & \\
\hline Sim & 78,4 & \\
\hline Síndrome demencial & & $<0,001$ \\
\hline Não & 55,2 & \\
\hline Sim & 100 & \\
\hline
\end{tabular}

Fonte: dados da pesquisa, 2013.
Tabela 5: Características associadas à perda de funcionalidade

\begin{tabular}{|c|c|c|}
\hline & \\
\hline & $\begin{array}{l}\text { Razão de risco } \\
\text { (IC95\%) }\end{array}$ & Valor $p$ \\
\hline Idade* & $1,002(1,0-1,005)$ & 0,05 \\
\hline Sexo* & $1,03(0,98-1,08)$ & 0,15 \\
\hline AVE prévio† & $0,95(0,90-1,00)$ & 0,06 \\
\hline $\mathrm{HAS}^{\star *}$ & $0,97(0,91-1,03)$ & 0,3 \\
\hline $\mathrm{DM}^{\star \star}$ & $1,06(0,99-1,12)$ & 0,05 \\
\hline $\begin{array}{l}\text { Insuficiência } \\
\text { cardíaca** }^{\star}\end{array}$ & $1,00(0,96-1,05)$ & 0,8 \\
\hline Demência** & $1,28(1,15-1,43)$ & $<0,001$ \\
\hline
\end{tabular}

Fonte: dados da pesquisa, 2013.

Nota: Regressão de Poisson modificada: * modelo 1: sexo, idade e demência, ${ }^{\dagger}$ modelo 2: modelo $1+$ AVE prévio, ** modelo 3: modelo $1+$ AVE prévio, HAS, DM, insuficiência cardíaca.

A partir disso, verificou-se que a incapacidade funcional é $28 \%$ maior naqueles idosos com diagnóstico de demência. A idade também foi identificada como fator de risco independente para perda de funcionalidade de forma significativa. Além disso, houve tendência em demonstrar que a presença de diabetes também está associada à perda funcional.

\section{Discussão}

Em relação às características sociodemográficas dos idosos institucionalizados, neste estudo observou-se que a média de idade ficou acima dos 85 anos. Os idosos, em sua maioria, apresentavam baixa escolaridade, e eram mulheres, considerando, ainda, $60 \%$ de viúvos(as). Nossos achados são semelhantes aos encontrados pela Sociedade Americana de Geriatria (SAG), que indica que quase metade das pessoas que vivem em resi- 
denciais geriátricos tem 85 anos ou mais, e em sua maioria são mulheres $(72 \%)$ (AGS, 2012). Outro estudo realizado em quatro ILPIs de Brasília também evidenciou que a maioria dos moradores são mulheres $(78,3 \%)$, com idade superior a 75 anos (FERREIRA et al., 2014).

$\mathrm{Na}$ análise dos diagnósticos de enfermagem, foi observado que mais de um terço da amostra apresentava nutrição desequilibrada e constipação. Outro estudo que analisou diagnósticos de enfermagem em idosos de uma unidade geriátrica da cidade de São Paulo verificou uma prevalência maior para nutrição desequilibrada (44,7\%) e menor para constipação $(22,3 \%)$ (SAKANO; YOSHITOME, 2007).

Para corroborar esse contexto nutricional, $25 \%$ dos idosos avaliados em nosso estudo foram classificados como desnutridos. O desequilíbrio nutricional no idoso está reconhecidamente relacionado ao aumento da mortalidade, à susceptibilidade a infecções e à redução da qualidade de vida (FÉLIX; SOUZA, 2009). Sabe-se também que a prevalência de desnutrição é maior em pacientes idosos institucionalizados do que em idosos que vivem em comunidade. Assim, os residentes em ILPIs tornam-se um grupo de risco para instalação ou agravamento de doenças e incapacidades (HERNANDEZ et al., 2007).

Ainda na análise das questões alimentares, verificou-se que $75 \%$ dos idosos alimentavam-se por via oral, exclusivamente, no entanto, mais de a metade dos residentes apresentavam déficit de deglutição, muitas vezes em função do quadro cognitivo debilitado, necessitando de monitoramento respiratório permanente, pois os riscos de desenvolver aspiração de conteúdo alimentar são aumentados. Para reforçar esses achados, constatou-se também uma prevalência de $56,6 \%$ de infecção respiratória recorrente.

As infecções do trato respiratório apresentam grandes taxas de morbidade e mortalidade no idoso, principalmente em idosos longevos e com presença de outras comorbidades (VILA-CORCOLES et al., 2008). Em países desenvolvidos, mais de $50 \%$ das internações por pneumonias são de idosos. A prevalência de infecção respiratória também é maior em idosos institucionalizados do que entre aqueles que vivem em comunidade (PILOTTO et al., 2009).

Quando observamos as comorbidades clínicas mais prevalentes nos residentes, constatamos que $58,3 \%$ eram hipertensos, $55,8 \%$ apresentavam insuficiência cardíaca e mais de um quarto dos idosos eram diabéticos ou sofreram AVE prévio. Outras pesquisas também apontam essas doenças como as principais comorbidades encontradas em residentes de ILPIs (SUZUKI; DEMARTINI; SOARES, 2009; LOURENÇO; VERAS; RIBEIRO, 2008).

No que diz respeito às características funcionais dos residentes, este estudo constatou que $85 \%$ dos idosos eram acamados ou necessitavam de dispositivo de auxílio para deambular. Esse fato ajuda a explicar o pequeno percentual de indivíduos considerados independentes para realização de atividades básicas da vida diária, apenas 10,8\% da amostra.

Um estudo realizado com idosos institucionalizados na cidade de Pelotas 
encontrou um percentual de idosos independentes de $20,6 \%$. O mesmo estudo aborda a dificuldade em comparar esse domínio com outros achados, uma vez que esse tipo de levantamento ainda é escasso (DEL DUCA et al., 2011).

A avaliação funcional foi definida como uma tentativa sistematizada de mensurar os níveis nos quais uma pessoa se enquadra, em uma variedade de áreas, tais como: integridade física, qualidade da automanutenção, qualidade no desempenho dos papéis, estado intelectual, atividades sociais, atitudes em relação a si mesmo e estado emocional (OLIVEIRA et al., 2006).

Um estudo demonstrou que os idosos com incapacidade funcional para as atividades básicas apresentam maior probabilidade de serem institucionalizados, levantando a hipótese da associação com o quadro de declínio cognitivo dos residentes de ILPIs (DEL DUCA et al., 2012). Outro achado também aponta a perda cognitiva como fator preponderante para institucionalização (STEPHAN et al., 2014). Há, ainda, evidencias do impacto positivo das intervenções de enfermagem, por meio de oficinas de reabilitação cognitiva, na preservação da funcionalidade e do autocuidado do idoso com demência (LINDOLPHO; SÁ; CRUZ, 2010).

Além disso, observamos que 57,5\% dos idosos apresentavam diagnóstico de quadro demencial, fator preponderante para explicar o grande percentual de incapacidade funcional. A SAG relata que em torno de $50 \%$ a $70 \%$ dos idosos institucionalizados apresentam quadro demencial, $25 \%$ dos residentes dependentes necessitam de ajuda para uma ou duas ABVDs, e $75 \%$ para três ou mais, dados que também vão ao encontro de nossos achados (AGS, 2012). Também foi diagnosticada a prevalência de incontinência urinária e fecal próxima a $90 \%$, fator que colabora diretamente para uma redução da qualidade de vida (DELARMELINDO et al., 2013) e para uma menor pontuação no índice de Katz. Conforme apresentado na Tabela 5 , a presença de quadro demencial e a idade foram considerados fatores de risco independentes para presença de incapacidade funcional.

Cabe ressaltar que a amostra conta com uma grande prevalência de idosos com quadro demencial e incapacidade funcional, limitado à análise, além do efeito de causalidade reversa, que dificulta o estabelecimento da temporalidade dos fatos e é inerente aos estudos transversais. Sugere-se a realização de novos estudos, preferencialmente com presença de grupo controle.

\section{Conclusões}

O presente estudo, conduzido com idosos institucionalizados, demonstrou uma alta prevalência de diagnósticos de enfermagem e de comorbidades clínicas, sobretudo com potencial impacto sobre a independência e a funcionalidade. Por meio de análise multivariada, identificou-se que idade e síndromes demenciais são fatores de risco independentes para incapacidade funcional. Dessa forma, adiciona-se dados epidemiológicos para o planejamento de avaliações de enfermagem em uma população ainda pouco estudada. 


\section{Nursing diagnoses in institucionalized older adults and the association with functional disability}

\section{Abstract}

The main objectives were to investigate the most prevalent nursing diagnoses related to institutionalized elderly and the implications on disability. A cross-sectional study was conducted. Data from all 120 residents of a nursing home were collected from June 2008 to March 2013. A standardized questionnaire was used to collect data about previous and present medical history. Functional status was obtained through the Katz Index Scale and respondents were categorized in independent or dependent. The association between nursing diagnosis and functional status was analyzed. A positive association was found between the presence of dementia and dependence $(p<0.001)$. Multivariate analysis demonstrated that dementia is an independent risk factor for functionality loss, and the presence of disability is $28 \%$ higher in elderly patients with dementia when compared to those without this condition $(p<0.001)$. Age was also identified as an independent risk factor for disability $(p=0,005)$. Concluding, nursing diagnoses are a fundamental part of this assessment and its use is of great importance to detect physiological and behavioral changes in these individuals, in addition to assisting in the planning and preparation of the plan of care.

Keywords: Geriatrics. Nursing Homes. Health Services for Older Adults. Disability. Nursing diagnoses.

\section{Referências}

AGS - AMERICAN GERIATRICS SOCIETY - Foundation for Health in Aging. Aging in the Know. Nursing Home Care. Disponível em: <http://www.nlm.nih.gov>. Acesso em: 23 set. 2012.

BARBOSA, B. R. et al. Avaliação da capacidade funcional dos idosos e fatores associados à incapacidade. Ciência e Saúde Coletiva, Rio de Janeiro, v. 19, n. 8, p. 3317-3325, ago. 2014.

BRASIL. Ministério da Saúde. Secretaria de Vigilância em Saúde. Departamento de Análise de Situações de Saúde. Plano de ações estratégicas para o enfrentamento das doenças crônicas não transmissíveis: 20112022. Brasília: Ministério da Saúde, 2011.

CAMARANO, A. A. (Org.). Cuidados de longa duração para a pessoa idosa: um novo risco social a ser assumido?. Rio de Janeiro: IPEA, 2010.

CARPENITO-MOYET, L. J. Diagnóstico de enfermagem: aplicação à prática clínica. 13 . ed. Porto Alegre: Artes Médicas, 2011.

DELARMELINDO, R. C. A. et al. Between suffering and hope: rehabilitation from urinary incontinence as an intervening component. Ciência e Saúde Coletiva, Rio de Janeiro, v. 18, n. 7, p. 1981-1991, jul. 2013.

DEL DUCA, G. F. et al. Incapacidade funcional em idosos institucionalizados. Revista Brasileira de Atividade Física e Saúde, Pelotas, v. 16, n. 2, p. 120-124, 2011.

DEL DUCA, G. F. et al. Indicadores da institucionalização de idosos: estudo de casos e controles. Revista de Saúde Pública, São Paulo, v. 46, n. 1, p.147-153, fev. 2012.

FÉLIX, L. N.; SOUZA, E. M. T. Avaliação nutricional de idosos em uma instituição por diferentes instrumentos. Revista de $\mathrm{Nu}$ trição, Campinas, v. 22, n. 4, p. 32-39, 2009. 
FERREIRA, L. S. et al. Perfil cognitivo de idosos residentes em instituições de longa permanência de Brasília - DF. Revista Brasileira de Enfermagem, Brasília, v. 67, n. 2, p. 247-251, 2014.

HERNANDEZ, A. G. et al. Estado nutricional de ancianos cubanos atendidos en 3 escenarios diferentes: comunidad, servicio de geriatría, hogar de ancianos. ALAN - Archivos Latinoamericanos de Nutricion, Caracas, v. 57, n. 3, p. 266-272, Sept. 2007.

IBGE - INSTITUTO BRASILEIRO DE GEOGRAFIA E ESTATÍSTICA. Censo demográfico 2010. Disponível em: <http://www.ibge. com.br>. Acesso em: 20 ago. 2012.

KATZ, S. et al. Studies of illness in the aged, the index of ADL: A standardized measure of biological and psychosocial function. JAMA - The Journal of the American Medical Association, Chicago, v. 185, p. 914-919, 1963.

LINDOLPHO, M. C.; SÁ, S. P. C.; CRUZ, T. J. P. Estimulação cognitiva em idosos com demência: uma contribuição da enfermagem. Revista Brasileira de Ciências do Envelhecimento Humano, Passo Fundo, v. 7, n. 1, p. 125-131, jan./abr. 2010.

LOURENÇO, R. A.; VERAS, R. P.; RIBEIRO, P. C. C. Confiabilidade teste-reteste do ini-exame do estado mental em uma população idosa assistida em uma unidade ambulatorial de saúde. Revista Brasileira de Geriatria e Gerontologia, Rio de Janeiro, v. 11, n. 1, p. 24-29, jan./abr. 2008.

MEIRA, E. C. et al. Condições de saúde e doença vivenciadas por idosos doentes e/ou fragilizados em processo de cuidado. InterScientia, João Pessoa, v. 1, n. 1, p. 111-123, 2013.

NANDA INTERNATIONAL - North American Nursing Diagnosis Association. Diagnóstico de enfermagem da Nanda: definições e classificação. Porto Alegre: Artmed, 2012.

OLIVEIRA, D. L. C.; GORETTI, L. C.; PEREIRA, L. S. M. O desempenho de idosos institucionalizados com alterações cognitivas em atividades de vida diária e mobilidade: estudo piloto. Revista Brasileira de Fisioterapia, São Carlos, v. 10, n.1, p. 23-29, 2006.

PASINATO, M. T. M.; KORNIS G. E. M. Ainserção dos cuidados de longa duração para idosos no âmbito dos sistemas de seguridade social: experiência internacional. In: CAMARANO, A. A. (Org.). Cuidados de longa duração para a pessoa idosa: um novo risco social a ser assumido?. Rio de Janeiro: IPEA, 2010. p. 39-66.

PILOTTO, A. et al. The multidimensional prognostic index predicts short- and longterm mortality in hospitalized geriatric patients with pneumonia. The Journals of Gerontology Biological Sciences \& Medical Sciences - series A, Washington, v. 64, n. 4, p. 880-887, Aug. 2009.

SAKANO, L. M.; YOSHITOME, A. Y. Diagnóstico e intervenções de enfermagem em idosos hospitalizados. Acta Paulista de Enfermagem, São Paulo, v. 20, n. 4, p. 495-498, out./dez. 2007.

SUZUKI N. M.; DEMARTINI S.M.; SOARES E. Perfil do idoso institucionalizado na cidade de Marília: subsídios para elaboração de políticas de atendimento. Revista de Iniciação Científica da FCC-UNESP, Marília, v. 9, n. 3, p. 256-268, 2009.

SCHMIDT, M. I. et al. Doenças crônicas não transmissíveis no Brasil: carga e desafios atuais. The Lancet, London, v. 5, p. 61-74, May 2011. Disponível em: <http://download. thelancet.com/flatcontentassests/pdfs/brazil/ brazilpor4.pdf>. Acesso em: 12 nov. 2014.

STEPHAN, A. et al. Older persons with dementia at risk for institutionalization in eight European countries: a cross-sectional study on the perceptions of informal caregivers and healthcare professionals. Journal of Advanced Nursing, Malden, v. 7, n. 4, p. 1-13, Aug. 2014.

VILA-CORCOLES, A. et al. Epidemiology of community-acquired pneumonia in older adults: a population-based study. $R e$ spiratory Medicine, Philadelphia, v. 18, n. 2, p. 309-316, Feb. 2008. 